\title{
Consistency between motor activity and perceived direction of rotation
}

\author{
CLARKE A. BURNHAM: \\ UNIVERSTY OI TEXAS. AUSTIN
}

\begin{abstract}
Three experiments were conducted to test the proposition that engagement in motor activity in a giren direction favors the perception of stimulus movement consistent with that direction. Ss simultaneously turned a crank and viewed a stimulus capable of apparent reversal of direction. Experiments $I A$ and $I B$ demonstrated that the perceived intial direction of rotation was more stable when it was consistent with the motor activity of the viewer than when it was inconsistent. Experiment 2 demonstrated that when Ss were instructed to perceive a particular direction of rotation for a period of time they tended to engage in motor activity consistent with that direction. The results were interpreted as supporting an efference theory of perception.
\end{abstract}

Objects rotating in depth frequently appear to reverse direction or undergo other transformations of apparent motion (Kenyon, 1898). Day and Power's (1965) analysis of rotary motion in depth, as amended by Hershberger (1967), specifies the stimulus conditions necessary for apparent reversal of rotating figures, namely a similarity of projected motion for clockwise and counterclockwise rotation. However, this theory does not specify the conditions under which a particular direction of rotation will be seen.

The problem investigated in the present study is the relationship between the perceived direction of rotation of an object capable of apparent reversal and the movements of an observer who is turning a crank which rotates the object. The general hypothesis of the investigation is that there is a consistency between motor activity and perception so that, for example, clockwise turning of the crank is associated with seeing clockwise rotation.

This hypothesized linkage of perception and motor activity stems from the efferent readiness theory of perception proposed by Festinger, Burnham, Ono, and Bamber (1967). This theory states that the conscious experience of perception is determined by the efferent readiness activated by the afferent visual stimulation. Efference theory has been directly tested by showing that the learning of a new afferent-efferent association for hand movements results in adaptation to prismatically induced curvature (Festinger et al, 1967) and the learning of a new afferent-efferent association for the extraocular muscles results in a decrement of the Muller-Lyer illusion (Burnham, 1968; Festinger, White, \& Allyn, 1968). Instead of studying the effect of learning a new afferent-efferent association, this study is concerned with the linkage between ongoing motor activity and perception. If what one sees is a function of how one is prepared to respond, then it is plausible that what one sees is also a function of ongoing motor activity, especially if the visual stimulation is ambiguous. Efference issued concurrently with afference may well determine the resulting perception.

There are a few published reports demonstrating that the perception of rotating figures can be manipulated, although the mechanisms which produced the changes in perception in these studies are not well understood. Power (1965) found that Ss instructed to report apparent reversals saw more reversals than Ss instructed to report nonreversals, a result attributed to reinforcement from fulfilling the reporting instructions. Mulholland (1963) found that the perception of ambiguous rotating figures could be manipulated by instructional viewing sets. Howard (1961) found that the direction and reversal rate of a rotating wire frame cube could be altered by prior exposure to the cube, which he attributed to satiation. The motor activity of the viewer was not manipulated in these studies, although subjective reports by Ss indicated that reversals of perspective of such figures as a three-dimensional Necker cube could be accomplished by altering the point of fixation (Uirich \& Ammons, 1959). In a study similar to the present investigation, Shopland and Gregory (1964) showed that active touch of a luminous wire frame cube reduced. but did not prevent, reversals of orientation. This result may retlect the influence of efference on the visual perception of anbiguous stimuli. However, it is also possible that other factors such as cognitive expectancies, covering parts of the cube with the hand, or differences in the way the cube was fixated when held vs only looked at were responsible for the effect.

Two different experimental manipulations were used in this investigation to explore the relationship between motor activity and the perception of rotary direction. The hypothesis of the first experiment (Experiment 1) was: the perception of the initial direction of rotation of figures capable of changing in apparent direction of rotation will be more stable if it is consistent with the motor activity of the $S$ than if it is inconsistent. This hypothesis was tested with two different stimuli. The stimulus for Experiment $1 \mathrm{~A}$ was a wire frame truncated pyramid; for Experiment $1 \mathrm{~B}$ the stimulus was a two-dimensional solid ellipse. The hypothesis of Experiment 2 was: when $S$ is directed to turn an ambiguous rotating object to make it continuously appear to rotate in a particular direction, the direction in which he turns the crank will tend to be consistent with the desired direction of rotation. This hypothesis was tested with the wire frame truncated pyramid

\section{EXPERIMENT 1A}

\section{Apparatus}

The apparatus for Experiment 1A was designed to allow the Ss to rotate the stimulus by turning a crank in a direction which $\mathrm{E}$ could make either consistent or inconsistent with the apparent direction of rotation of the stimulus. The stimulus was located at one end of a wooden frame and turned by a crank positioned in front of $S$ which he turned with his right hand. Both the crank and stimulus rotated in a horizontal plane. The gear linkage between the crank and the rotating object was capable of being changed by $\mathrm{E}$ between trials so that clockwise $(\mathrm{CW})$ rotation of the crank could produce either $\mathrm{CW}$ or counterclockwise $(\mathrm{CCW})$ rotation of the object. The $S$ viewed the stimulus with his right eye througlı a $3 / 16$ in. diameter hole with his chin and forehead resting against a padded frame to minimize head movements. The $S$ 's right eye was located approximately $3 \mathrm{in}$. in front of the hole, the center of rotation was $52-1 / 2 \mathrm{in}$. from the hole, and the background, a homogeneous gray, was located $7 \mathrm{in}$. behind the center of rotation. The center of rotation of the crank and stimulus object were aligned with S's right eye, and the center of the stimulus was at eye level.

The dependent variable for Experiment IA was the time from the onset of rotation to the first perceived alteration in rotation. The $S$ reported when the stimulus altered its direction of rotation by removing his left hand from a pressure sensitive switch which he depressed when he started turning the crank. Closure of this switch operated a stop-clock.

The stimulus for Experiment $1 \mathrm{~A}$ was a truncated wire frame pyramid constructed from $3 / 32$ in. rods and painted flat black. The large base was a 3 in. square, the small base a $1-1 / 8$ in. square. and the altitude was $2-1 / 4 \mathrm{in}$. A shaft attached to the middle of one side of the large base rotated the pyramid. The pyramid was oriented so that its bases were perpendicular to the plane of rotation.

When stationary and oriented with the planes of the bases 
perpendicular to the line of sight, the small base of the truncated pyramid can be seen in front of at the same distance as, or behind the large base although it is easier to perecive the small base in front of the large regardless of their true positions. During rotation the apparent direction of rotation alternates; this is accompanied by a reversal in the perspective of the bases. The perceived initial direction of rotation of the truncated pyramid is controlled by the perceived orientition of the bastes. It is veridical if the perception of the stationary figure is veridicial. However, if the perception of the stationary figure is illusory. the initial perception of rotation will also be illusory. When the small hase is actually behind and is seen as being in front. actual ('W rotation will appear as $C C W$ rotation.

\section{Design}

A total of 48 undergraduate students fulfilling an introductory psychology course requirement participated in the study. They were randomly assigned to the experimental conditions. Half the Ss turned the crank (W: the other half turned it CCW. Two Es each tested half the Ss in each turning direction group. In addition to these between-subject variables there were two within-subject variables: startine oricntation and consistency vs inconsistency between perecived and turning directions of rotation. For half the trials the starting orientation was normal (small base actually near and perceived as near) and for half it was reversed (small base actually away and perceived as near). On half the trials the initially perceived direction of rotation was consistent with the turning direction and on the other hatf it was inconsistent. This was manipuated by a combination of the starting orientation and the gear linkage. Each of the 24 Ss in each turning condition was presented with a different secuence of the four possible combinations of starting oricntation and initial apparent direction of rotution. The sequence was presented once for four practice trials during which t turned the crank and repeated twice for the cight experimental trials during which $S$ turned the crank.

\section{Procedure}

The Ss were told that the small base would be positioned nearer to them at the beginning of each trial. Practice sessions ensured that Ss could make this interpretation of the figure regardless of its actual position. At the beginning of each rotation trial Ss were asked if the small base appeared nearer. Those few Ss who responded negatively or equivocally were told to try to see it that way and were able to do so. The practice rotation trials were designed to acquaint Ss with the types of perceptual changes they might see (reversal and oscillation) and to provide experience with the operation of the switch. During these trials $E$ turned the crank at a rate of approximately $60 \mathrm{rpm}$; Ss were instructed to turn it at the same rate. A trial was terminated after $90 \mathrm{sec}$ of rotation if alteration of the direction of rotation had not occurred by then. It was necessary to terminate an experimental trial for only one $S$, once on a consistent trial and once on an inconsistent trial.

\section{Results}

It was hypothesized that if the initially perceived direction of rotation was consistent with the direction in which the object was turned, perception of this direction would be more stable than if the apparent direction of rotation and the turned direction were inconsistent. The data analyzed were the averages of the times to reversal for each combination of the two within-subject variables (normal vs reversed starting orientation and consistency vs inconsistency of initially perceived direction of rotation and turning direction). These times for the two within-subject variables and the two between-subject variables (CW vs CCW turning of the crank and $E_{1}$ vs $E_{2}$ ) were analyzed by a four-way analysis of variance. The only significant effect was for the consistency variable $(\mathrm{F}=4.77, \mathrm{df}=1 / 44, \mathrm{p}<.05)$. The perception of the initial direction of rotation was more stable when it was consistent with the motor activity of the $\mathrm{S}$. When consistent, the mean time to reversal was $9.80 \mathrm{sec}$; when inconsistent it was $8.62 \mathrm{sec}$. None of the other main effects and none of the interactions approached statistical significance.

\section{EXPERIMENT $1 \mathrm{~B}$}

\section{Apparatus}

Experiment $1 \mathrm{~B}$ was a replication of Experiment $1 \mathrm{~A}$ with a change in the stimulus and with minor design and procedural changes. The experimental hypothesis, the apparatus, and the dependent variable were the same.

The stimulus for Experiment $1 \mathrm{~B}$ was an ellipse, with a major axis of $3 \mathrm{in}$. and a minor axis of $3 / 4 \mathrm{in}$. It was cut from $.029 \mathrm{in}$. sheet metal and painted flat black. The ellipse was rotated about its minor axis by a shaft affixed to the bottom edge.

When a rotating ellipse is viewed monocularly, the perceived direction of rotation is correct for approximately one-half. the trials and incorrect for the others. However, when viewed binocularly the direction of rotation is veridical for most Ss. An additional viewing hole was provided for the S's left eye to permit binocular viewing. The $\mathbf{E}$ could block the perception of the ellipse through this left eye viewing hole by sliding a screen between it and the ellipse.

\section{Design}

The Ss used in this study were 30 different Ss from the same pool as that used for Experiment 1A. Six Ss were not run in the experiment since they were unable to meet a criterion of errorless perception in an initial binocular test for veridical perception of rotation. As in the previous study, half the Ss turned the crank $\mathrm{CW}$ and the other half turned it $\mathrm{CCW}$. For half the trials the initial direction of rotation was consistent with the hand movement and for the others it was inconsistent. Twn of the $12 \mathrm{Ss}$ in each turning condition were presented with on if the six possible sequences of two consistent and two inconsistent trials. The sequence of trials was repeated once for the eight experimental trials.

\section{Procedure}

After the viewing holes were adjusted, the binocular test for veridical perception of rotary direction was conducted. The E turned the ellipse $360 \mathrm{deg}$ four times and requested Ss to state the direction of rotation each time. The Ss then turned it four times and reported the direction. Only Ss with errorless performance in this test continued in the experiment.

There were four practice trials to provide experience with the desired $60 \mathrm{rpm}$ rotation rate, the use of the timing switch, and perception of the possible alterations of rotation which included reversal. oscillation, apparent expansion and contraction of the sides of the ellipse. and a flapping of the sides about the axis of rotation. The $\mathrm{E}$ turned the crank for the first two practice trials; the Ss operated it for the second two. The practice trials were followed by the eight experimental trials. The Ss viewed the cllipse binocularly for the first revolution of all trials so the initial perception of rotary direction would correspond to the desired experimental treatment. After the first revolution. E blocked the left eye view of the ellipse. A trial was terminated after $45 \mathrm{sec}$ if alteration of the direction of rotation had not occurred by then. All Ss saw reversal within this period during the experimental trials.

\section{Results}

As in the truncated pyramid study the data analyzed were the times to initial reversal or alteration of rotation from the onset of rotation. The average times for the four consistent and the four inconsistent trials were analyzed with the between-subject variable of $C W$ vs CCW turning of the crank by a two-way analysis of variance. When the initial direction of rotation was consistent with the turning direction, the mean time to reversal was $7.68 \mathrm{sec}$. when inconsistent it was $6.78 \mathrm{sec}$. The difference between these times is significant $(\mathrm{F}=4.40, \mathrm{df}=1 / 22, \mathrm{p}<.05)$. In this study there was also a significant effect for turning direction $(F=6.45$, $\mathrm{d} f=1 / 22, \mathrm{p}<.05)$. The times to reversal were longer $(8.29 \mathrm{sec})$ for those Ss who turned the crank CCW than for those who turned it CW (6.17 sec). This may reflect a slower rate of turning for the CCW Ss. However. the comparable data for Experiment IA are in the opposite direction; time to reversal was longer although not 
significantly so, for those Ss who turned the crank CW. Consequently, this effect is not seadily interpretable. There was no interaction between the turning direction variable and the consistency variable.

\section{Discussion}

The results of Experiments IA and IB support the experimental hypothesis; the perception of the initial direction of rotation of figures capable of changing in apparent direction of rotation is more stable if the perceived direction is consistent with the direction in which $S$ is turning a crank connected to the object. The replication of the phenomenon with differences in stimuli, Ss, Es, and with somewhat different experimental procedures increases the generality of the results. Further support for the hypothesis that what one does affects what one sees would be obtained by varying the experimental task. This was done in Experiment 2.

\section{EXPERIMENT 2}

In Experiment 1 the Ss perceived a certain initial direction of rotation which was either consistent or inconsistent with the direction in which they turned the crank. In Experiment 2 the Ss were asked to turn the crank so that the stimulus would always appear to turn in a particular direction. $C W$ rotation. With monocular viewing of the truncated pyramid, $C W$ rotation could be perceived during either $C W$ or $C C W$ turning of the crank. After an $S$ started turning $C W$ to perceive $C W$ rotation, a shift in the perspective of the bases would produce apparent $C \mathrm{CW}$ rotation. S would then have to turn the crank CCW to produce the required appearance of $\mathrm{CW}$ rotation. It was hypothesized that $\mathrm{Ss}$ would turn the crank more often in a CW direction to sec C.W rotation since the motor activity and the pesception would then be consistent. The control for this crank turing contition was a motor condition in which the Ss performed the same task of making the stimulus appear to rotate $C W$ by controlling the direction in which a motor turned the stimulus. Since the efference of the Ss on these trials was not directly related to the rotary motion of the stimulus. no preference for $\mathrm{CW}$ or $\mathrm{CCW}$ turning was expected. In Experiment 2 data on the crank turning rate and the frequency of apparent reversals were obtained as well as the data necessary to test the experimental hypothesis, the duration of actual $\mathrm{CW}$ and $\mathrm{CCW}$ rotation of the stimulus during the crank and motor trials.

\section{Apparatus}

The apparatus used in Experiment 1 was modified slightly for Experiment 2. Two stimuli were presented simultaneously. One, whose direction of rotation was unambiguous, was a cylinder 3 in. in diameter and 3 in. high divided into four alternating black and gray quadrants. This striped cylinder was rotated $\mathrm{CW}$ at a constant speed of $60 \mathrm{rpm}$ by a motor. The other stimulus was the wire frame truncated pyramid used in Experiment lA. For the crank trials this was rotated by $S$ turning a crank as in Experiment 1 . The gear linkage was arranged so $\mathrm{CW}$ cranking produced $\mathrm{CW}$ rotation. For the motor trials the pyramid was rotated by a $60 \mathrm{rpm}$ instantly reversible motor. The motor direction was controlled by a switch located to the S's right. The up position of the switch produced $\mathrm{CW}$ rotation and the down position $\mathrm{CCW}$ rotation.

The stimuli were located 12 in. apart with the pyramid on the left. They were equidistant from the S's right eye and 55 in. in front of a $1 / 4 \times 2$ in. viewing slit through which they were viewed monocularly with the right eye. A red fixation point was provided on the white background which was $91 / 2$ in. behind the stimuli and directly in front of the viewing slit.

The durations of actual $\mathrm{CW}$ and $\mathrm{CCW}$ rotation of the pyramid were recorded on stop-clocks and the number of reversals of direction and the number of revolutions made during the trials were recorded on counters. Timing apparatus started and stopped the cylinder for trials of 1 min with an intertrial interval of $45 \mathrm{sec}$.

\section{Design}

Eighteen undergraduate students fulfilling an introductory psycholosy course requirement participafed in the shidy. All Ss were run in both crank and motor trials. Half were run in a crank-motor secluence and half in a motot-crank secfucnce. There were two crank and two motor practice trials followed by four crank and four motor experimental trials. The data analyzed to test the effect of efference on perception were the differences between the duration of $C W$ and $C C W$ rotation of the pyramid for the crank and motor experimental trials.

\section{Procedure}

The Ss were told that the cylinder would be rotated $C W$ by a motor and that their task would be to control the direction of rotation of the pyramid so that it appeared to turn in the same direction as the cylinder turned. The phenomenon of apparent reversal of the pyramid and the technique for achieving apparent CW movement with the crank and motor were demonstrated. The Ss practiced turning the crank $\mathrm{CW}$ and $\mathrm{CW}$ and operating the motor in both directions while viewing the pyramid binocularly.

At the beginning of each trial, Ss were asked if the small basc of the pyramid appeared nearer than the large base (which was the veridical orientation) and then told to fixate the dot and be prepared to start turning the pyramid as soon as the cylinder started rotating. Because of the perceived orientation of the pyramid and the Ss' knowledge of the crank linkage and the motor switch positions, all Ss started turning the pyramid in a CW direction.

The turning rate for the crank trials was controlled by instructing $S s$ to turn the pyramid at about the same rate as the cylinder turned and, after each practice trjal, te]ling those $S$ s who turned it slower than 50 rpm or faster than 70 rpm to speed up or slow down.

\section{Results}

The difference between the duration of CW and C CW rotation for the crank and motor experimental trials was analyzed with the sequence variable by a two-way analysis of variance. The data analyzed were virtually equivalent to the simpler measure of the difference between the duration of C.W rotation for the two types of trials. The reason for subtracting the duration of CCW rotation from the duration of $\mathrm{CW}$ rotation from each trial is that the total turning times do not sum to $1 \mathrm{~min}$. Because of time spent making the reversals and hesitations the total times averaged $57.55 \mathrm{sec}$ for the crank trials and $57.48 \mathrm{sec}$ for the motor trials. The only significant effect was for the crank-motor variable $(F=12.78$, $\mathrm{df}=1 / 16, \mathrm{p}<.01)$. When the Ss controlled the pyramid with the crank, they turned it $\mathrm{CW}$ for an average of $59.2 \%$ of each $1 \mathrm{~min}$ trial; when controlling its direction with the motor, they turned it $\mathrm{CW}$ an average of $53.7 \%$ of each trial. $\mathrm{CW}$ perception of rotation was more strongly associated with the issuance of efference for $\mathrm{CW}$ turning than with operating a motor to turn in a CW direction.

The mean turning rate for the four experimental crank trials was $61.75 \mathrm{rpm}$ with .95 confidence limits of 58.54 to 64.96 . The correlation between the turning rate and the duration of $\mathrm{CW}$ turning was not significant $(r=.07, d f=16)$; the correlation between turning rate and the number of perceived reversals was also not significant $(\mathrm{r}=.17 \mathrm{df}=16)$. Within the range of turning rates obtained in this study there was no tendency for the actual number of rotations to be associated with either the duration of CW turning or the number of perceived reversals.

The Ss saw more reversals of direction when lusning the pyramid by hand than when it was tumed by the motor $(\mathrm{F}=12.68, \mathrm{df}=1 / 16, \mathrm{p}<.01)$. There were an average of 20.4 reversals during each crank trial and 15.5 reversals during each motor trial. It is possible that the less smooth rotation accompanying hand turning created stimulus conditions which facilitate the perception of oscillation. The correlation between the number of reversals and the duration of CW turning was not significant for either the hand trials $(r=42, \mathrm{df}=16)$ or the motor trials $(r=.06, \mathrm{df}=16)$. 


\section{DISCUSSION}

The results of Experiment 2 further support the hypothesized linkage between perception and motor activity. The Ss achieved consistency between what they saw and what they did by turning the crank CW more often than CCW. Thus not only is the perception of rotary direction more stable when the motor activity corresponds to the perception, but there is also a tendency to perform motor activity which is consistent with a desired perception.

These experiments were based on the efferent readiness theory of perception. Before further considering the relationship between the experimental results and this theory, it would be worthwhile to consider other possible explanations of the obtained results.

The results are clearly not caused by a learned preference for perceiving rotation in one direction rather than another. The difference between consistent and inconsistent trials in Experiments $1 \mathrm{~A}$ and $1 \mathrm{~B}$ was of the same magnitude regardless of whether the perceived starting direction of rotation was $\mathrm{CW}$ or $\mathrm{CCW}$. Nor are the results attributable to visual clues resulting in a tendency to perceive the direction of rotation veridically rather than illusorily. The consistency effect in Experiment $1 \mathrm{~A}$ was obtained when the actual direction of rotation differed from the perceived direction (on the trials when the starting orientation of the pyramid was reversed) as well as when the two corresponded (normal starting orientation).

It is equally difficult to explain the results in terms of cognitive sets or expectation. It might be argued that Ss expect $\mathrm{CW}$ rotation to be associated with $\mathrm{CW}$ turning of the crank and the consistency effect to result from the cognition that there is something unusual when $\mathrm{CW}$ rotation is associated with $\mathrm{CCW}$ turning. However, the Ss in Experiments 1A and 1B perceived a direction of movement different from their turning direction at the beginning of half of the trials, including the practice trials. Thus the possibility that the unusualness of the inconsistency between the perception and the motor activity produced the consistency effect was minimized. The Ss in Experiment 2 knew that the up position of the motor switch, like $\mathrm{CW}$ turning of the crank, caused actual $\mathrm{CW}$ rotation of the pyramid. This cognitive knowledge did not prevent these Ss from reversing the switch position and seeing what they knew to be actual CCW rotation as CW rotation for an average of $46.3 \%$ of motor trials.

Satiation is frequently invoked as an explanation for alterations in the perception of ambiguous figures (e.g., Howard, 1961; Orbach, Ehrlich, \& Heath, 1963). It is unclear what predictions would be made from a satiation hypothesis of figural reversal to account for the finding that there is a consistency between motor activity and perception. In fact, if cross-modal satiation were to occur-if the effect of turning a crank $\mathrm{CW}$ were to sum with the effect of perceiving an object rotate in a $\mathrm{CW}$ direction-more rapid reversals might be expected in the consistent conditions of Experiments $\mathrm{IA}$ and $\mathrm{IB}$ and more ${ }^{\prime} \mathrm{CW}$ cranking than $\mathrm{CW}$ cranking might be expected in Experiment 2.

The results are consistent with the efferent readiness theory of perception, although they are not able to be derived from it. In Experiments $\mathrm{IA}$ and $\mathrm{BB} \mathrm{E}$ controlled the efference issued to the arm by instructing the Ss to turn the crank in a particular direction. A perception, presumably resulting from the activation of efferent readiness, which was consistent with this motor activity was more stable because of the simultaneous issuance of consistent efference. In Experiment 2 the S's task was to perceive a particular direction of rotation which was accompanied by the issuance of efference to the arm consistent with the desired perception. The simultaneous issuance of consistent efference facilitated the desired perception of the ambiguous stimulus. In sum, these studies have demonstrated that what one is doing at the time of impinging visual stimulation influences how the pattern of stimulation from an ambiguous rotating object is seen. Further research is necessary to test directly the efferent readiness theory of perception by showing that how one is prepared to respond determines the perceived direction of rotation of ambiguous stimuli.

\section{REFERENCES}

BURNHAM, C. A. Decrement of the Müller-Lyer illusion with saccadic and tracking eye movements. Perception \& Psychophysics, 1968, 3, 424-426.

DAY, R. H., \& POWER, R. P. Apparent reversal (oscillation) of rotary motion in depth: An investigation and a general theory. Psychological Review, 1965, 72, 117-127.

FESTINGER, L., BURNHAM, C. A., ONO, H., \& BAMBER, D. Efference and the conscious experience of perception. Journal of Experimental Psychology, Monograph Supplement, 1967, 74, (4, Whole No. 637).

FESTINGER, L., WHITE, C. W., \& ALLYN, M. R. Eye movements and decrement in the Müller-Lyer illusion. Perception \& Psychophysics, 1968 , 3, 376-383.

HERSHBERGER, W. A. Comment on "Apparent reversal (oscillation) of rotary motion in depth." Psychological Review, 1967, 74, 235-238.

HOWARD, I. P. An investigation of a satiation process in the reversible perspective of revolving skeletal shapes. Quarterly Journal of Experimental Psychology, 1961, 13, 19-33.

KENYON, F. C. A curious optical illusion connected with an electric fan. Science, $1898,8,371-372$.

MULHOLLAND, T. Instructional sets and motion perceived while viewing rotating stimulus objects. Journal of Psychology, 1963, 56, 233-237.

ORBACH, J., EHRLICH, D., \& HEATH, H. A. Reversibility of the Necker cube: I. An examination of the concept of "satiation of orientation." Perceptual and Motor Skills, 1963, 17, 439-458.

POWER, R. P. The effect of instructions on the apparent reversal of rotary motion in depth. Quarterly Journal of Experimental Psychology, 1965, 17, 346-350.

SHOPLAND, C. D., \& GREGORY, R. L. The effect of touch on a visually ambiguous threc-dimensional figure. Quarterly Journal of Experimental Psychology, 1964, 16, 66-70.

ULRICH, $P_{,}$\& AMMONS, R. B. Voluntary control over perceived dimensionality (perspective) of three-dimensional objects. Proceedings of the Montana Academy of Science, 1959, 19, 169-173.

\section{NOTES}

1. Robert Hardin assisted the author in running Experiment 1A; Kent Johnson conducted Experiment 1B; and Leonard Robertson conducted Experiment 2.

2. Address: Department of Psychology, Mezes Hall, University of Texas, Austin, Texas 78712 .

(Accepted for publication July 9, 1968.) 